\title{
द

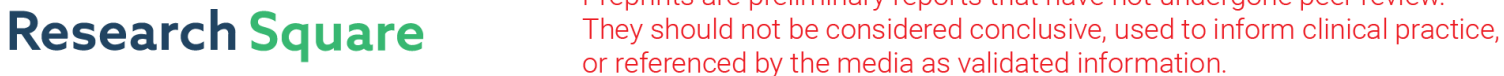

\section{Are tagged fish like others? Insights from growth and stress physiological profile in two marine fish species}

\section{Sébastien Alfonso}

COISPA Tecnologia \& Ricerca - Stazione Sperimentale per lo Studio delle Risorse del Mare

\section{Walter Zupa}

COISPA Tecnologia \& Ricerca - Stazione Sperimentale per lo Studio delle Risorse del Mare

\section{Amedeo Manfrin}

Istituto Zooprofilattico Sperimentale delle Venezie

\section{Eleonora Fiocchi}

Istituto Zooprofilattico Sperimentale delle Venezie

\section{Maria Dioguardi}

Universita degli Studi di Palermo Dipartimento di Scienze della Terra e del Mare

\section{Mariano Dara}

Universita degli Studi di Palermo Dipartimento di Scienze della Terra e del Mare

\section{Matteo Cammarata}

Universita degli Studi di Palermo Dipartimento di Scienze della Terra e del Mare

\section{Giuseppe Lembo}

COISPA Tecnologia \& Ricerca - Stazione Sperimentale per lo Studio delle Risorse del Mare

Pierluigi Carbonara ( $\square$ carbonara@coispa.it )

COISPA Tecnologia e Ricerca Stazione Sperimentale per lo Studio delle Risorse del Mare https://orcid.org/0000-0002-2529-2535

\section{Short communication}

Keywords: Accelerometer tags, Telemetry, Welfare, Stress, Sparus aurata, Dicentrarchus labrax, Aquaculture

Posted Date: May 12th, 2020

DOI: https://doi.org/10.21203/rs.3.rs-23507/v2

License: (c) (1) This work is licensed under a Creative Commons Attribution 4.0 International License. Read Full License 


\section{Abstract}

Background: Telemetry applied to aquatic organisms has recently developed greatly. Physiological sensors have been increasingly used as tools for fish welfare monitoring. However, for the technology to be used as a reliable non-invasive welfare indicator, it is important that the tagging procedure does not disrupt fish physiology, behaviour and performance. In this communication, we share our medium-term data on stress physiological profile and growth performance after tag implantation in two important marine fish species for European aquaculture, the sea bream (Sparus aurata ) and the European sea bass ( Dicentrarchus labrax).

Results: Blood samples after tag implantation (46 days for the sea bream and 95 days for the sea bass) revealed no differences between tagged and untagged fish in cortisol, glucose and lactate levels, suggesting that the tag implantation does not induce prolonged stress in these species. Moreover, the specific growth rates were similar in the tagged and untagged fish of both species.

Conclusion: Tag implantation does not have medium-term consequences for the stress physiology and growth performance of these two marine fish species in a controlled environment. These observations support the use of accelerometer tags as valuable tools for welfare monitoring in aquaculture conditions, as they do not affect the fish's welfare and health. This study also shows that tagged fish can be sampled during experiments and considered a representative portion of the population, as they display growth and physiological parameters comparable to those of untagged fish.

\section{Introduction}

Over the past decades, telemetry applied to aquatic organisms has greatly developed in terms of tag miniaturization, battery life, software and hardware [1]. These tags are precious tools for the characterization and monitoring of behaviour in a wide range of organisms, including fish [2]. Moreover, electronic tags can also be equipped with environmental sensors that can record diverse data, such as temperature, depth and salinity, while monitoring physiological parameters, such as heart and ventilation rates or muscle activity [3-6]. Although these physiological sensors have mainly been used in the wild in the context of conservation and ecology, they have progressively been employed in aquaculture, serving as welfare indicators of common stressors (e.g. slaughtering practices, water quality and stocking density) $[4,7-9]$.

Telemetry studies assume that tagged fish are physiologically representative of the entire population. Therefore, it is essential that the tag does not negatively affect growth performance, physiology and survival. The implantation method and site and the tag's size are important factors for preventing the disruption of the physiological state, normal movement, and growth performance of tagged fish [10-13] and avoiding bias in the collected data. The maximum tag weight generally considered acceptable is no more than $2 \%$ of the fish's body weight in air (the so-called " $2 \%$ rule") [10-12]. However, in some cases, the " $2 \%$ rule" is not enough to avoid negative effects on the fish's health and welfare, such as stress, 
inflammation or obstruction of internal organs, or on its buoyancy and swimming performance [10]. Most of our knowledge about the link between tag implantation and stress is based on salmonids; therefore, more species-specific information is needed.

In this study, we collected data from two different experiments, on the European sea bass (Dicentrarchus labrax) and the sea bream (Sparus aurata), two of the most important species for European aquaculture $[14,15]$, aiming to evaluate growth performance and the physiological stress profile of tagged fish at least 46 days after implantation. Their physiological stress profile was assessed by comparing the means of plasma stress indicator values (cortisol, glucose and lactate levels) with those of untagged fish, while growth was assessed by comparing the specific growth rates (SGR) between tagged and untagged fish.

\section{Methods}

\section{Animals}

Sea breams (mean weight \pm SD: $314.6 \pm 49.1 \mathrm{~g}$ ) were obtained from the commercial hatchery Ittica Caldoli (Lesina, Italy). After three weeks of acclimation, ID100 radio frequency identification (RFID) tags (Trovan, Netherlands) were implanted in the fish, which were then separated into three fiberglass tanks of $1.2 \mathrm{~m}^{3}(\mathrm{n}=115$ fish per tank; 30 $\mathrm{kg} / \mathrm{m}^{3}$ ), forming triplicates. The fish were reared in marine water at a constant temperature of $18{ }^{\circ} \mathrm{C}$, salinity of $35 \mathrm{PSU}$ and a pH of 7.1. The water was completely replaced three times a day, and the oxygen levels were continuously monitored by an automatic system programmed to maintain the dissolved oxygen concentration above $5 \pm 1$ ppm.

European sea bass fish (mean weight \pm SD: $335.5 \pm 62.4 \mathrm{~g}$ ) were obtained from the commercial hatchery Panittica Pugliese SpA (Torre Canne, Italy). After three weeks of acclimation, RFID tags (ID100) were implanted in the fish, which were then separated into three fiberglass tanks of $1.2 \mathrm{~m}^{3}$ ( $\mathrm{n}=35$ fish per tank; $\sim 10 \mathrm{~kg} / \mathrm{m}^{3}$ ), forming triplicates. The fish were left undisturbed for two months before the start of the experiment. The water parameters (temperature, salinity and oxygen) were constant and similar to those for the sea breams.

Throughout the experimental period, all fish were exposed to a 12L:12D photoperiod and were fed 1\% of their body mass using commercial feed (Skretting Marine 3P, Italy) 
dispensed by automatic feeders for $3 \mathrm{~h}$ every morning.

\section{Experimental procedure}

At the beginning of the experiment $\left(t_{0} ;\right.$ Fig. 1$)$, the fish were gently removed from their rearing tanks and anaesthetized with a hydroalcoholic clove oil solution (30 mg/L) [16, 17]. Morphometric parameters (body weight and total length) were recorded to calculate the SGR (see the "Growth measurements and SGR calculations" section).

\section{Tag implantation}

At the beginning of the experiment (Day 0) for sea bass and 18 days later for sea breams (Day 18) (Fig. 1), V9AP acoustic accelerometer tags (Vemco Systems Inc., Nova Scotia, Canada) were implanted in nine randomly selected sea bass and five randomly selected sea breams (at least two fish from each tank, except one fish from one tank for the sea bream experiment), as described in Carbonara et al. [7]. Briefly, the fish were subjected to fasting for $24 \mathrm{~h}$ before implantation and were anaesthetized using a hydroalcoholic clove oil solution in doses of $30 \mathrm{mg} / \mathrm{L}[16,17]$. The transmitter was inserted into the body cavity through a 1.5-cm incision. The incision was then carefully sutured, and the fish were injected with antibiotic (sodic ampicillin-cloxacillin; $1 \mathrm{mg} / \mathrm{kg} 24 \mathrm{~h}^{-1}$ ) [18] before being returned to their home tanks until the end of the experiment $\left(t_{1} ;\right.$ Fig. 1$)$. The mean tag weight accounted for $1.63 \% \pm 0.32$ and $0.90 \% \pm 0.21$ of the sea bream and sea bass body mass, respectively. All tagged fish recovered within a few days, and no mortality linked to the surgical procedure was observed [7]. To evaluate possible tag effects, 12 untagged sea breams and 9 untagged sea bass were randomly selected as controls (at least three fish per tank; Table 1) and were monitored during the experimental period.

Table 1 Sample sizes and mean masses of tagged and untagged sea breams and European sea bass

\begin{tabular}{|c|c|c|c|c|}
\hline Species & Status & $\mathbf{N}$ & Mass at $t_{0}(g)$ & Mass at $t_{1}(g)$ \\
\hline \multirow[t]{2}{*}{ Sea bream (Sparus aurata) } & Tagged & 5 & $312.6 \pm 48.2$ & $407.8 \pm 52.4$ \\
\hline & Untagged & 12 & $309.4 \pm 65.3$ & $389.5 \pm 90.8$ \\
\hline \multirow{2}{*}{ European sea bass (Dicentrarchus labrax) } & Tagged & 9 & $423.8 \pm 80.7$ & $466.9 \pm 79.5$ \\
\hline & Untagged & 9 & $425 \pm 76.4$ & $479.2 \pm 71.4$ \\
\hline
\end{tabular}

$t_{0}$ : beginning of experiment; $t_{1}$ : end of experiment 


\section{Growth measurements and SGR calculations}

At $t_{1}$ (Days 46 and 95 after tagging the sea breams and sea bass, respectively; Fig. 1), the tagged and untagged fish were once again gently removed from their rearing tanks and anaesthetized with clove oil solution as described above. Their body weight was measured (in grams) to calculate the differences in SGR between $t_{0}$ and $t_{1}$. The SGR was calculated according to the following equation [19]:

[Please see the supplementary files section to view the equation.]

where $W$ is the total weight at the end $\left(t_{1}\right)$ and the beginning of the experiment $\left(t_{0}\right)$, and $T$ is the number of feeding days between $t_{0}$ and $t_{1}$.

\section{Blood sampling and stress indicator analysis}

After the morphometric measurements (2-3 minutes after anaesthesia inducement), blood samples of $0.5 \mathrm{~mL}$ were immediately taken from the first branchial arch of the tagged and untagged fish using a heparinized syringe. The samples were then centrifuged at 15,000 g for $3 \mathrm{~min}$, and plasma was collected and stored at $-20{ }^{\circ} \mathrm{C}$ until further processing, described below.

The plasmatic cortisol, glucose and lactate concentrations were measured as described in Carbonara et al. [7]. Briefly, the cortisol concentration was determined using solid-phase competitive chemiluminescent enzyme immunoassays with a cobas Cortisol II kit (Roche, Switzerland). The glucose and lactate concentrations were determined using kits $17630 \mathrm{H}$ and 17285 (Sentinel Diagnostics, Italy), respectively, based on the enzymatic colorimetric Trinder reaction (GOD/PAP for glucose and PAP for lactate).

\section{Statistical analysis}

Statistical analyses were performed using the R software version 3.6.2 [20] at a 95\% level of significance. Homoscedasticity of the data was a priori tested using the Shapiro-Wilk test. The appropriate statistical test (either the Wilcoxon test or the $t$-test) was then 
performed to compare the SGRs and physiological stress indicators (cortisol, glucose and lactate) between the tagged and untagged fish of each species.

\section{Results}

In terms of growth performance, the SGR was similar between the tagged and untagged fish for both the sea bream $(W=38, p=0.44)$ and the sea bass $(t=-0.58, \mathrm{p}=0.56$; Fig. 2$)$ between $\mathrm{t}_{0}$ and $\mathrm{t}_{1}$, which correspond to a period of 64 days for the sea breams and 95 days for the sea bass.

At $t_{1}$, the plasma concentrations of stress indicators were overall similar between the tagged and untagged fish of both species (Fig. 3). More specifically, the plasma cortisol concentration showed no statistically significant differences either in the sea breams $(W=32, p=0.88)$ or in the sea bass $(t=0.94$, $p=0.36$; Fig. 3a). The levels of the secondary stress indicators (i.e. glucose and lactate) were also similar both in the sea breams ( $W=25.5, p=0.67$ for glucose and $t=1.04, p=0.33$ for lactate) and in the sea bass ( $W=39, p=0.93$ for glucose and $t=1.18, p=0.26$ for lactate; Figs. $3 \mathrm{a}, 3 \mathrm{~b}$ ).

\section{Discussion}

Our results show that after a relatively long period (46 days for the sea bream and 95 days for the sea bass) following surgical implantation of accelerometer tags, the tagged fish were comparable with the untagged fish in terms of both growth and stress physiology in aquaculture conditions. To our knowledge, this is the first report on the sea bream and the European sea bass, two important species for European marine aquaculture. These findings support the use of physiological tags in these two species in aquaculture conditions.

Surgical implantation of accelerometer tags is perceived as a stressor for fish, causing cortisol release into the blood [21], which is the main stress hormone in teleost fishes [22]. It is a relatively acute response of organisms coping with stressors before regaining homeostasis, but it may last only a few days, depending on the species. For instance, in rainbow trout (Oncorhynchus mykiss), a heart rate increase was observed during the first $72 \mathrm{~h}$ following surgical implantation of a heart rate sensor, after which it was stabilized [23], suggesting that fish regain homeostasis relatively quickly after this stressful event. Jepsen et al. [21] reported similar observations in Chinook salmon, where physiological stress indicators were higher up to $24 \mathrm{~h}$ following tag implantation but were comparable with those of untagged fish at most seven days later. In our experiments, 46 and 95 days after tag implantation in sea breams and sea bass, respectively, the levels of all monitored stress indicators (cortisol, glucose and lactate) were found to be similar to those of untagged fish and consistent with the literature regarding these species [7, 24]. Our results confirm that tag implantation does not induce chronic stress in either the sea bream or the sea bass, as observed in various other fish species $[21,25]$. It is thus important to emphasize that tag implantation does not exert long-term adverse effects on a high-stress responder species such as the European sea bass [26-28]. 
Nonetheless, although we did not directly investigate the acute stress response to tag implantation by measuring physiological stress indicators after the surgical procedure, we did observe that generally, the tagged fish did not eat for two to four days post-operatively (personal observations), probably because of surgery-induced stress. Indeed, stress and growth are closely related; stress is known to inhibit food intake and, consequently, limit the energy available for biological processes, including growth [29]. Therefore, it appears that acute stress is indeed induced by tag implantation, but it only lasts a few days in these species. Moreover, this period of no food intake has no long-term consequences on growth, as shown by the similar SGRs between the tag and untagged fish of both species. It has been demonstrated in different fish species that when the " $2 \%$ rule" is applied, growth performance is generally not impacted $[12,21,30]$. The similar growth rates between tagged and untagged fish can be explained by compensatory growth, which is a period of unusually rapid growth following a period of undernutrition [31]. It is noteworthy that we observed similar growth rates between the tagged and untagged fish in two different stocking densities ( $10 \mathrm{~kg} / \mathrm{m}^{3}$ for the sea bass and $\sim 30 \mathrm{~kg} / \mathrm{m}^{3}$ for the sea bream), which suggests that tagged fish can compensate growth and continue their normal life under different rearing conditions.

In conclusion, surgical implantation of accelerometer tags does not cause medium-term changes in the stress physiological profile and growth of either sea breams or sea bass reared in a controlled environment. Future studies are needed to investigate exactly how long these species take to recover from stress induced by tag implantation and thus be considered "normal" fish, displaying normal behaviour (e.g. feeding) and basal levels of stress indicators. Our study confirms (i) that accelerometer tags are useful tools for welfare monitoring, as they do not affect the welfare and health of tagged fish and (ii) that tagged fish can be sampled during experiments and considered representative of the population, as they display growth and physiological parameters comparable to those of untagged fish.

\section{Declarations}

\section{Ethics approval and consent to participate}

The sea bream experiment was performed in accordance with Italian national legislation (Legislative Decree 26/2014) and EU Directive 2010/63/EU with authorization from the Italian Ministry of Health (No. $665 / 2016-P R)$. The sea bass experiment was performed in add date of Italian national legislation, however the experiment was done in accordance with European Commission recommendation 2007/526/EC C(2007) 2525 and EU Directive 2010/63/EU. In both experiments, all fish recovered fully from the tag implantation procedures, and no associated mortality was observed.

\section{Consent for publication}

Not applicable.

\section{Availability of data and materials}


The datasets used and/or analysed during the current study are available from the corresponding author on reasonable request.

\section{Competing interests}

The authors declare that they have no competing interests.

\section{Funding}

This study was funded by ANIHWA ERA-Net (WIN-FISH), the Italian Ministry of Health (Department for Public Veterinary Health, Nutrition and Food Safety - Id Project - 54 Welfare, Health and Individuality in Farmed FISH) and Ritmare SP2_WP4_A23_U003.

\section{Authors' contributions}

WZ and PC performed the tag implantations in both species. PC, WZ, EF, AM, MDa, MDi, MC and PL performed the blood sampling and the analysis of physiological parameters. SA performed the statistical analyses and prepared the figures. PC and SA wrote the first draft of the manuscript. All authors read and approved the final manuscript.

\section{Acknowledgements}

Not applicable.

\section{References}

1. Hussey NE, Kessel ST, Aarestrup K, Cooke SJ, Cowley PD, Fisk AT, et al. Aquatic animal telemetry: A panoramic window into the underwater world. Science. 2015;348:1255642.

2. Cooke SJ, Hinch SG, Wikelski M, Andrews RD, Kuchel LJ, Wolcott TG, et al. Biotelemetry: a mechanistic approach to ecology. Trends Ecol Evol. 2004;19:334-43.

3. Bjarnason A, Gunnarsson A, Árnason T, Oddgeirsson M, Sigmarsson AB, Gunnarsson Á. Validation of ECG-derived heart rate recordings in Atlantic cod (Gadus morhua L.) with an implantable data logging system. Anim Biotelemetry. BioMed Central; 2019;7:1-10.

4. Carbonara P, Scolamacchia M, Spedicato MT, Zupa W, Mckinley RS, Lembo G. Muscle activity as a key indicator of welfare in farmed European sea bass (Dicentrarchus labrax L. 1758). Aquac Res. 2015;46:2133-46.

5. Millidine KJ, Metcalfe NB, Armstrong JD. The use of ventilation frequency as an accurate indicator of metabolic rate in juvenile Atlantic salmon (Salmo salar). Can J Fish Aquat Sci. 2008;65:2081-7.

6. Metcalfe JD, Wright S, Tudorache C, Wilson RP. Recent advances in telemetry for estimating the energy metabolism of wild fishes. J Fish Biol. 2016;88:284-97.

7. Carbonara P, Alfonso S, Zupa W, Manfrin A, Fiocchi E, Pretto T, et al. Behavioral and physiological responses to stocking density in sea bream (Sparus aurata): Do coping styles matter? Physiol Behav. 
Elsevier; 2019;212:112698.

8. Brijs J, Sandblom E, Axelsson M, Sundell K, Sundh H, Huyben D, et al. The final countdown: Continuous physiological welfare evaluation of farmed fish during common aquaculture practices before and during harvest. Aquaculture. 2018;495:903-11.

9. Carbonara P, Alfonso S, Gai F, Gasco L, Palmegiano G, Teresa M, et al. Moderate stocking density does not influence the behavioural and physiological responses of rainbow trout (Oncorhynchus mykiss) in organic aquaculture. 2020; https://doi.org/10.1111/are.14640.

10. Jepsen N, Schreck C, Clements S, Thorstad EB. A brief discussion on the $2 \%$ tag/bodymass rule of thumb. Aquat Telem Adv Appl Proc Fifth Conf Fish Telem held Eur. 2005;295.

11. Jepsen N, Koed A, Thorstad EB, Baras E. Surgical implantation of telemetry transmitters in fish: how much have we learned? Hydrologia. 2002;483:239-48.

12. Smircich MG, Kelly JT. Extending the $2 \%$ rule: The effects of heavy internal tags on stress physiology, swimming performance, and growth in brook trout. Anim Biotelemetry. 2014;2:1-7.

13. Cooke SJ, Graeb BDS, Suski CD, Ostrand KG. Effects of suture material on incision healing, growth and survival of juvenile largemouth bass implanted with miniature radio transmitters: case study of a novice and experienced fish surgeon. J Fish Biol. 2003;62:1366-80.

14. FAO. The State of Fisheries and Aquaculture in the world 2018 - Meeting the sustainable development goals. 2018.

15. Vandeputte $M$, Gagnaire $P$-A., Allal F. The European sea bass: a key marine fish model in the wild and in aquaculture. Anim Genet. 2019;50;195-206.

16. Zupa W, Carbonara P, Spedicato MT, Lembo G. Modelling swimming activities and energetic costs in European sea bass (Dicentrarchus labrax L., 1758) during critical swimming tests. Mar Freshw Behav Physiol. https://doi.org/10.1080/10236244.2015.1073456

17. Iversen M, Finstad B, McKinley RS, Eliassen RA. The efficacy of metomidate, clove oil, Aqui-S ${ }^{T M}$ and Benzoak ${ }^{\circledR}$ as anaesthetics in Atlantic salmon (Salmo salar L.) smolts, and their potential stressreducing capacity. Aquaculture. 2003;221:549-66.

18. Lembo G, Carbonara P, Scolamacchia M, Spedicato MT, Bjørnsen JE, Holand B, et al. Introduction of a new physiological acoustic electromyogram transmitter. Fish Manag Ecol. 2008;15:333-8.

19. Hopkins KD. Reporting Fish Growth: A Review of the Basics. J World Aquac Soc. 1992;23:173-9.

20. R Core Team. R: A Language and Environment for Statistical Computing. Vienna, Austria; 2015. /

21. Jepsen N, Davis LE, Schreck CB, Siddens B. The Physiological Response of Chinook Salmon Smolts to Two Methods of Radio-Tagging. Trans Am Fish Soc. 2011;130:495-500.

22. Sadoul B, Geffroy B. Measuring cortisol, the major stress hormone in fishes. J Fish Biol. 2019;94:540-55.

23. Brijs J, Sandblom E, Rosengren M, Sundell K, Berg C, Axelsson M, et al. Prospects and pitfalls of using heart rate bio-loggers to assess the welfare of rainbow trout (Oncorhynchus mykiss) in aquaculture. Aquaculture; 2019;509:188-97. 
24. Carbonara P, Dioguardi M, Cammarata M, Zupa W, Vazzana M, Spedicato MT, et al. Basic knowledge of social hierarchies and physiological profile of reared sea bass Dicentrarchus labrax (L.). PLoS One. 2019;1-16.

25. Rożyński M, Kapusta A, Demska-Zakęś K, Hopko M, Sikora A, Zakęś Z. The effects of surgically implanted dummy tags on the survival, growth performance, and physiology of pikeperch (Sander lucioperca). Fish Physiol Biochem. 2017;43:999-1010.

26. Samaras A, Santo CE, Papandroulakis N, Mitrizakis N, Pavlidis M, Höglund E, et al. Allostatic load and stress physiology in European seabass (Dicentrarchus labrax L.) and gilthead seabream (Sparus aurata L.). Front Endocrinol. 2018;9:1-13.

27. Alfonso S, Sadoul B, Gesto M, Joassard L, Chatain B, Geffroy B, et al. Coping styles in European sea bass: The link between boldness, stress response and neurogenesis. Physiol Behav. 2019;207:7685.

28. Alfonso S, Sadoul B, Cousin X, Bégout M-L. Spatial distribution and activity patterns as welfare indicators in response to water quality changes in European sea bass, Dicentrarchus labrax Article. Appl Anim Behav Sci. 2020;104974.

29. Sadoul B, Vijayan MM. Stress and Growth. Fish Physiol. Elsevier Inc.; 2016. p. 167-205.

30. Anras MLB, Covés D, Dutto G, Laffargue P, Lagardère F. Tagging juvenile seabass and sole with telemetry transmitters: medium-term effects on growth. 2003;60:1328-34.

31. Dobson SH, Holmes RM. Compensatory growth in the rainbow trout, Salmo gairdneri Richardson. J Fish Biol. 1984;25:649-56.

\section{Figures}


(a)

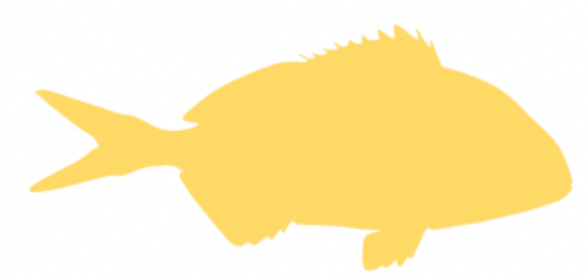

Sparus aurata

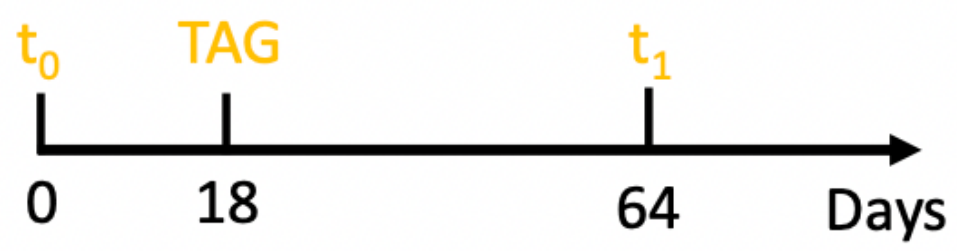

(b)

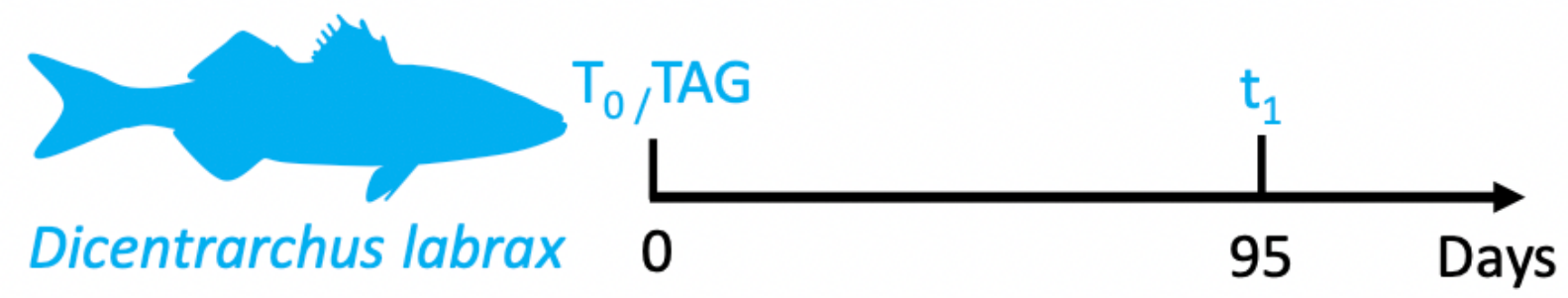

Figure 1

Time course schedule (days) of the experimental procedure for Sea bream (Sparus aurata; yellow) and European sea bass (Dicentrarchus labrax; blue). T0 and t1 represent the beginning and the end of the experiment, corresponding to the first and final measurement for SGR calculation. TAG represents the period of implantation of accelerometers tag. 

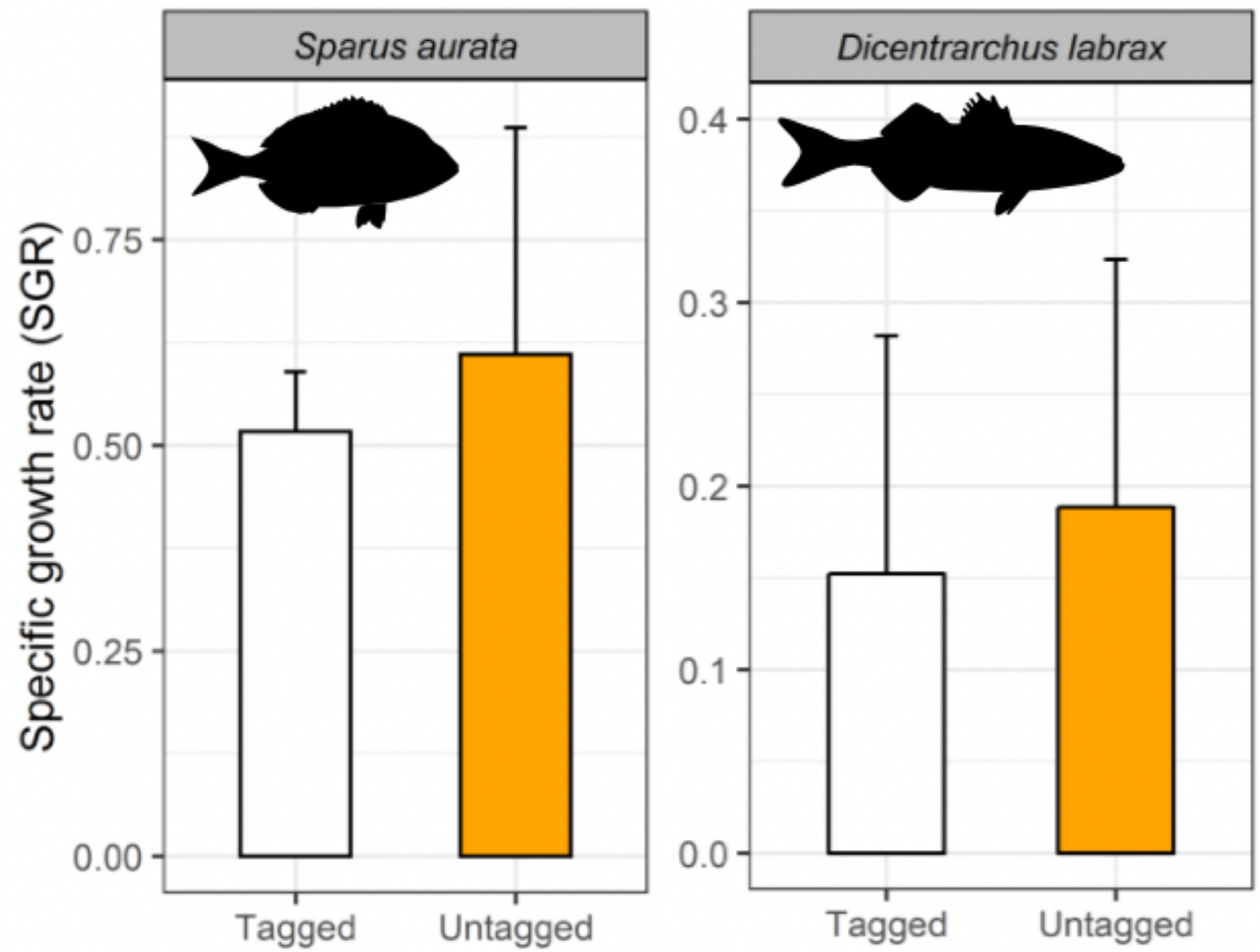

Figure 2

Specific growth rate (SGR) of untagged (white bars; $n=12$ sea bream and $n=9$ European sea bass) and tagged fish (orange bars; $n=5$ Sea bream and $n=9$ European sea bass). Values are mean \pm SD. See main text for statistics. 

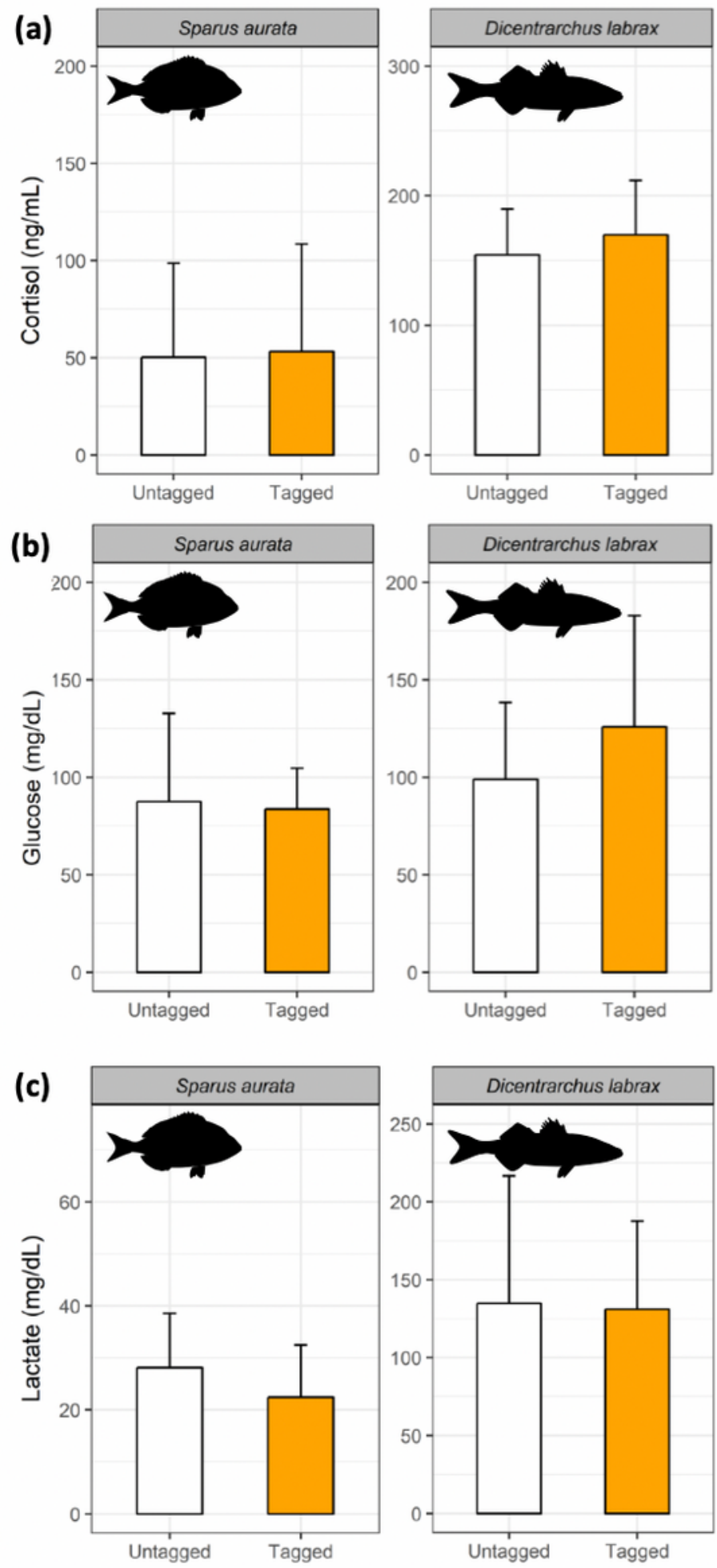

Figure 3

Stress physiological profile of untagged (white bars; $n=12$ sea bream and $n=9$ European sea bass) and tagged fish (orange bars; $n=5$ sea bream and $n=9$ European sea bass) at $t 1$. (A) Cortisol $(n g / m L),(B)$ Glucose $(\mathrm{mg} / \mathrm{dL})$ and $(C)$ Lactate $(\mathrm{mg} / \mathrm{L})$. Values are mean \pm SD. See main text for statistics.

\section{Supplementary Files}


This is a list of supplementary files associated with this preprint. Click to download.

- Equation.docx 